كلمة التحرير

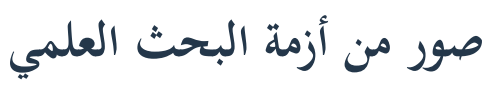

\section{إدارة التحرير}

قضايا المنهجية في الفكر الإسلامي قضايا متشعبة، وتتعلق بالتفكير والبحث، كما تتعلق بالتعامل مع النصوص والأفكار والأشياء والأحدث والأشخاص. وتميل الرؤية المعرفية إلى الواقع وقضاياه ومشكلاته إلى محاولة بناء علاقة بين مناهج التفكير أو مناهج البحث أو مناهج التعامل من جهة والصورة التي نجد عليها واقع الناس وأحوالهم من جهة أخرى. ومن ثمّ تصبح هذه العلاقة وسيلة لفهم الظواهر وتفسيرها، وتصبح دراسة قضايا المنهجية على هذا الأساس مهمة جداً في جهود الإصلاح. والأمر في هذا الشأن لا يقتصر على واقع المسلمين، بل هو أمر إنساني عام. لكن الفارق أن المجتمعات الأخرى تبدو أكثر وعياً على هلى القضايا المنهجية. وفي كلمة التحرير هذه مجموعة من الإشارات الموجزة إلى صور مختلفة من الأزمة القائمة في ميدان البحث العلمي في هذه المجتمعات الأخرى، تبين كيف يستشعر المعنيون بالبحث هذه الصور، لعلّ في ذلك ما يدفع قراءنا الأعزاء إلى تتبعها، وتتبّع تمثّالامّا في واقعنا الراهن، وحثّ الخطى على إيلاء الموضوع ما يجتاجه من اهتمام

$$
1 \text { تعليم مناهج البحث العلمي في العلوم الدينية. }
$$

تقدم معظم الجامعات ضمن برامجها للدراسات العليا مادة بعنوان "مناهج البحث" لتساعد الطلبة الباحثين على استكمال متطلبات البحث المطلوب منهم للحصول على الدرجة العلمية. ونظراً لأن معظم البحوث التي تتم في برامج التعليم الديني تستبعد مناهج البحوث الميدانية الكمية، المألوفة في معظم العلوم الإنسانية والاجتماعية، فإن الاهتمام ينصرف إلى الوراقيات والأدبيات الخاصة بموضوعات الدراسات الدينية؛ أي أن تدريب الطلبة ينصرف إلى كيفية تحديد المصادر والمراجع والحصول عليها وأخذ المعلومات منها. وحيث لا تتوافر كشافات وفهارس عامة بالبحوث والكتابات الخاصة حول الموضوعات المختلفة تيسر للباحث 
رصدها، وحيث لا تتوافر مواقع لإيداع البحوث تيسر للباحث الحصول عليها، يقع عبء كبير على مدرسي مادة مناهج البحث، وعلى موظفي الخدمات المكتبية لتيسير مهمة الباحث وإعانته.

ويبدو أن أزمة البحث في الدراسات الدينية -وبخاصة فيما يتعلق بتعليم مناهج البحث وبعدم كفاية الخدمات المكتبية- لا تقتصر على البلاد العربية والإسلامية، بل تتعدى ذلك إلى البلاد الغربية والولايات المتحدة على وجه التحديد. فالعلاقة بين "البحث" و "المكتبة" فيما يتعلق بالدراسات الدينية في الولايات المتحدة تواجه مشكلة مستعصية منذ ثلاثة عقود، وربما كانت هذه المشكلة انعكاساً لأزمة الهوية في المعرفة العلمية وعلاقتها بالرؤية الكلية السائدة في ميدان البحث. وهذا ما يراه باري هاملتون أحد الباحثين المتخصصين في الدراسات الدينية في مناقشته للموضوع في ورقة عرضها في المؤتمر السنوي "للاتحاد الأمريكي للمكتبات الثيولوجية" في حزيران 2003م . ويعزو الباحث المشار إليه أسباب الأزمة إلى نظرية المعرفة الوضعية المبنية على العلوم الطبيعية، تلك النظرية التي فشلت في توضيح موقف علم المكتبات وموقع البحث العلمي فيه، ومن هنا جاء تعليم مناهج البحث في الدراسات الدينية في إطار النموذج الوضعي الذي يُعلي من شأن المعلومات ويقلل من شأن التفسير. وكأن المعلومات هي لبنات بناء، والبحث هو جمعها في سلة. وهكذا فإن محتوى مادة مناهج البحث يركز على المهارات التقليدية لاستعمال المكتبة؛ أي نظام التصنيف، وأخذ الملحوظات، وطريقة التوثيق، وكأن المعرفة هي "شيء" ما يختلف عن الباحث؛ شيء يوجد في الكتاب والدوريات. أما المصلة النهائية لهذا النموذج من تعليم مناهج البحث فهو إنجاز مهمة كتابة مادة، وليس التطور والتحول في خبرات الباحث. والبحث في القضايا الدينية يتطلب مناهج معينة في فهم طبيعة المعرفة، لأن محتوى هذا البحث بطبيعته يتعامل مع العلاقة بين الإلهي والإنساني، ويتم في ظروف زمانية ومكانية محددة تتعلق بالإنسان، ولا يستطيع الباحثون في هذه الموضوعات أن يفسروا محتوى المصادر والمراجع المكتبية في معزل عن المعنى المشترك الذي يتطور في إطار مجتمع من الناس. إن البحث في القضايا الدينية هو دعوة للباحث للتأمل في خبراته حول رحلته في طريق الإممان والبحث عن الروح؛ ولذلك فإنه يبدأ من تقرير هوية الباحث المؤمن بالله الخالق وهذا ما يجعل البحث بحثاً دينياً، ولا يسمح الباحث في هذا الميدان بزرع إسفين يفصل بين التكوين الفكري 
والتكوين الروحي. وتتعزز أهمية هذه الملاحظة من وجود بعض الدراسات والبحوث الدينية التي توقع الطلاق

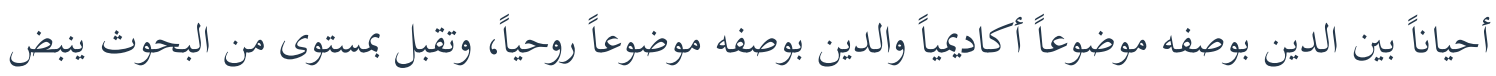
بالحياة الروحية، لكنه لا يمتلك إلاّ القليل من المستوى الأكاديمي.

ويصف هاملتون مساق مناهج البحث في الجامعات الدينية بأنه يقوم على المحاضرات، حيث يعِي المدرِّمُ أنواعَ الأدب الديني، وطرق استخدام المكتبة والقضايا الشكلية في إعداد البحث. وتعزّز هذه النظرية في تدريس المساق الفكرة النمطية بأن البحثَ عملٌ يقوم به الباحث بصورة منفردة، وأن الباحث المؤهل لا لا يحتاج إلى معونة من المدرس أو موظف المكتبة. وهذا المساق بصورته التقليدية في الجامعات الدينية يعرض البحث في خطوات محددة ومتتابعة: اختيار الموضوع، إيجاد المراجع، تلخيص النقاط المهمة منها في بطاقات،

Cyclical وكتابة النتائج. وفي مجال نقد هذا الواقع يؤكد الباحث أن عملية البحث ذات طبيعة دورية ويرفض النظرة التقليدية للبحث على أنه خطوات متسلسلة ذات بداية وهاية في كل خطوة. وتعكس طبيعة المواد التي تمت مراجعتها . المت

إن البحث الحقيقي أكثر بكثير من مجموع المراجع التي اعتمدها الباحث؛ إنه يعكس عملية التفكير المنظم الذي يكتشف ارتباطات قائمة بين الأشياء والأفكار ويخلق نظاماً جديداً في عالم المعرفة. هذه العملية تتضمن تفاعل مكونات عديدة في نفس الوقت منها: الغرض، والسياق المفاهيمي، وأسئلة البحث، وطرق التأكد من الصدق، حيث تتقارب الأفكار وتتباعد، وتتجاذب وتتنابذ، وتقنع وتثني عن الاقتناع. وقد يكون من المفيد في هذا المقام التأكيد على أن مادة مناهج البحث في أقسام الدراسات الدينية، تحتاج إلى جدية في التخطيط لها وتنفيذها بطريقة تتخلص فيها من النظرة الحالية إليها بوصفها متطلباً شكلياً لا يحمل أهمية خاصة، بل عليها أن تؤمن -والإيمان هدفها- بأن مادة مناهج البحث في الدراسات الدينية، وطريقة تدريسها تحتل موقعاً أساسياً في بنية هذه الدراسات ونموها وتطور مؤسساتا في الظروف التي يعيشها

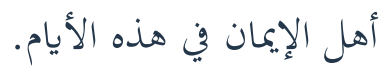

2. أزمة الخيار بين المناهج الكمية والنوعية سادت ميدان البحث العلمي في العلوم الإنسانية والاجتماعية معظم القرن العشرين مناهجُ بحثية تعتمد البيانات الكمية الميدانية والتحليل الإحصائي لهذه 
البيانات. وكان التنافس في كسب المنزلة العلمية ينصب على درجة التعقيد والتفصيل في طرق جمع البيانات الكمية وتحليلها، بصورة توحي بأن من لا يلجأ إلى هذه الأساليب المعقدة لا يكون معداً إعداداً جيداً في تئي أساليب البحث العلمي. ولكن هذه الصورة بدأت تتغير منذ بداية الربع الأخير من القرن العشرين. حيث أخذ عدد من العلماء يوجهون إلى البحوث الكمية الإحصائية نقداً شديداً، طال فلسفتها ومرجعيتها وطرق تفسير نتائجها. وكثر الحديث عن المناهج النوعية الكيفية بوصفها بديلاً أكثر ملاءمة للعلوم الإنسانية والاجتماعية، وأخذت هذه المناهج بتحد قبولاً في الدوائر الأكاديمية بصورة عامة.

ولكن الدراسة المتأنية لهذه الدراسات النوعية تكشف عن جذور معرفية ومنهجية للجدل الذي لا يزال قائماً لا ترتبط بالفرق بين طبيعة كل من الدراسات النوعية والكمية وأغراضها، وإنما ترتبط بسياسات البحوث ومصالح الفئات المعنية هذذه السياسات وتوجهاتا. فمثلاً يجد الأستاذ المساعد في الجامعات الذي لم يتم تثبيته في الخدمة صعوبة بالغة في شق طريقه إلى التثبيت إذا سلك في بحوثه المناهج النوعية، بينما تبقى ممارسة هذه المناهج ميزة لأولئك الذين بتحاوزوا هذه المرحلة . إن السعي إلى تأكيد مشروعية المناهج البحثية يشغل بال العديد من الباحثين. فالباحثون الذين يستخدمون أشكالاً ختلفة من البحوث النوعية مهتمون بتأكيد مشروعية عملهم. ويثير هذا الاهتمام ولو بصورة جزئية الجدل القائم حول طبيعة فهمنا البشري والكيفية التي نصل فيها إلى معرفة هذا العالم. ويف الظروف الطبيعية يقود هذا الجلدل إلى نقاشات ناقدة مفيدة تحسِّن من إجراء البحوث. ولكنَّ هذا الجدل يعكس في كثير من الأحيان من المعاني أكثر مما يبدو في ظاهر الأمر ـ ويخفى في طياته صراعاً سياسياً وتنافساً على المواقع الأكاديمية أو احتكاراً لها. وهكذا توصف بعض المناهج البحثية بأهما أفضل، وأدق وأكثر علمية، بينما هُمََُُّ مناهج بكثية أخرى وتوصف بأهما ضعيفة، صحفية، لا تستند إلى نظرية. الخ. وتشكل هذه الحقائق عقبات قوية تحول دون تقدم البحث باستخدام المناهج النوعية، وقليل من

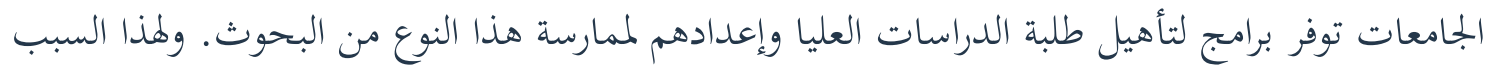
ليس من المستغرب أن ينظر إلى البحوث النوعية بوصفها ميزة محصورة على الأساتذة القدامى في هذا الجامعات. ويحدث هذا في الوقت الذي تبدو فيه الحاجة إلى البحوث النوعية قد وصلت إلى مستوى الأزمة؛ 
فالظروف المعقدة التي تتصف بسرعة التغيير في عالم اليوم تتطلب من الجامعات إعداد طلبة الدراسات العليا والباحثين، بحيث يكونوا قادرين على التعامل مع القضايا الراهنة في ميادين العمل بطريقة شمولية، تتجاوز المعرفة المزأة وتعبر خطوط التخصصات الضيقة .

إن الطبيعة الخاصة لبعض القضايا البحثية تحتاج إلى بحوث معمقة بتعل المناهج النوعية أفضل من غيرها لهذا الغرض. لكن ضغوط الأساتذة القدامى، وعدم توافر التدريب الكافي في البحوث النوعية وضغوط دقات الزمن التي تلح على الأساتذة الجلدد بتلبية متطلبات التثبيت في الخدمة أو الترقية بتعل كثيراً من الباحثين يلجيئون إلى البحوث الكمية التقليدية. 3 انعكاسات ما بعد الحداثة على مناهج البحث العلمي.

تمثل تشكيلات ما بعد الحداثة رؤية كلية إلى العالم، تنظر بعين الشك إلى جميع الرؤى الأخرى. وتشير هذه التشكيلات إلى المدارس والحركات والتوجهات الفكرية التي تمخضت عنها مرحلة ما بعد الحداثة التي نمر بها الآن وهي مذاهب تأتي وتذهب، لكن حالة ما بعد الحداثة جاءت لتبقى . وتشير أدبيات ما بعد الحداثة إلى الصورة التي تحاول رسمها للعالم، في ضوء تعريف الهوية بأها أمر يتم بناؤه وليس اكتشافه- من مصادر ثقافية متعددة، وأن فهم الأحكام القيمية والخلقية يتم على أساس الرؤى الكونية التي يتم بناؤها اجتماعياً. وتؤكد هذه الصورة على الإبداع والتنوع والسخرية والهزل في الفن والأدب، وتشدد على أهمية الوعي بأن جميع أنواع الحدود هي تركيبات اجتماعية للحقيقة يتم عبورها ومحوها وإعادة بنائها. ومن المهم أن ننبه إلى الانعكاسات الواسعة لهذه الخصائص على البحث العلمي، فالباحثون في مدارس ما بعد الحداثة يرون أن الظاهرة الإنسانية تتغير بمجرد دراستها، وبخاصة إذا كان المشاركون فيها يعرفون شيئاً عن دورهم في البحث، ويرون أن العلم ليس متحرراً من القيم، بل هو مشحون بقيم صريحة وضمنية، وبخاصة عندما يتعلق الأمر بنتائج العلم وتطبيقاته التكنولوجية، وأن سعي العلم ليس سعياً نهو اليقين؛ لأن اليقين في رأي ما بعد الحداثيين عبث لا طائل من ورائه، ولأن المعرفة محلية وليست كونية، ومشروع التنوير قد تم تفكيكه، والناس المختلفون في هذا العالم لا يفكرون بالطريقة نفسها، فكيف يعتقد الحداثيون بوجود طريقة عقلية واحدة؟ 
وقد طورت المدارس ما بعد الحداثية طرقاً في البحث تعتمد في مجملها المناهج النوعية، ومن بين هذه المناهج المرتبطة مباشرة بالفكر ما بعد الحداثي ما يسمى ب "تحليل النظم الهيولية" التي ظهرت في العقدين الآخيرين وأخذت تعرض أساليبها في وصف وفهم عمليات التغير والنمو والتطور ذات الطبيعة الهيولية غير المنتظمة في تشكلاتا؛ مثل أنماط المناخ والنظم البيئية وكثير من الظواهر التي تحدث بصورة غير خطية. وتنطلق منهجية تحليل النظم الهيولية من التشكيك في موقف الحداثيين القائل بأن الطبيعة ذات سلوك منتظم يمكن التنبؤ به، وبالتالي يمكن التحكم فيه. كما تنطلق هذه المنهجية من الاعتقاد بأن المهارات التي نجح الغرب فيها أيما نجاح هي التشريح والتفكيك واختزال المشكلات إلى أبسط مكوناتها حتى تصبح تافهة لا خير فيها، الأمر الذي يفترض البحث عن نظم في البحث تقدم ابتحاهاً جديداً نهو العمل المجدي، ويتشرب الفكر التطوري البنائي، الذي يتتبع الفوضى في الظواهر، ويبحث عن العلاقات غير الخطية، ويحاول تعريف الأنماط والصور والتنظيم الذاتي وخصائص التكيف للعمليات المعقدة التي توظف الرياضيات غير الخطية في التعبير عن التمثيلات غير الكمية في هذه الظواهر، وهذا ما تقدمه منهجية تحليل النظم الهيولية.

إن كثيراً من علماء ما بعد الحداثة التفكيكيين يشعرون بأن العلم الحديث يجب التخلص منه تماماً. بينما يقترح علماء ما بعد الحداثة التركيبيون أن هذا العلم إذا أعيد تركيبه بطريقة مناسبة ووضع في سياق جديد، فإن الطريقة العلمية يمكنها أن تزود الباحثين بأدوات قوية ومفيدة لفهم الأحداث، التي بدوها لا يملكون إلا المراوغة لتجنب بحثها. كذلك فإن القول بأن العلم الحديث هو تبسيط زائد لا علاقة له بالعالم الخارجي لا يمكن أن ينظر إليه نظرة جديّة، وعلى العكس من ذلك فإن المعرفة التي تشتق من العلوم الحديثة قدمت مقاربات عديدة للحقيقة الحسية، وقد لا تكون هذه المقاربات تامة من بعض النواحي، لكنها مقاربات ذات

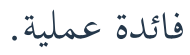

أمّا مدرسة ما بعد الحداثة في أقل تعبيراها تطرفاً، فإنها تتوقع من المؤسسات العلمية وطرق الحصول على المعرفة وبناء الحقيقة أن تتغير، بحيث يجسر العلمُ الهوَّة بين الحداثة وما بعد الحداثة، وضمن هذا الإطار فإنه يمكن فهم النظريات والقوانين والنماذج على أغها تعبيرات مجازية لا تمثل معرفة ثابتة، ولكنها تمثل حالة "سائلة" من الأفكار تتطور وتتقدم نتيجة لمعالجات الباحثين. وضمن عملية إعادة التركيب هذه فإن منهجية العلوم الإنسانية يمكنها أن تأخذ صورة من الوصف الاجتماعي التفاعلي. 
4. أزمة في أخلاقيات البحث العلمي ثمة صور من التحيز في الممارسات البحثية ونتائجها، تمثل بعض

وجوه الأزمة في البحث العلمي. ومن ذلك ما يتعلق بالمشكالات الأخلاقية التي تثيرها عقود التعاون بين المؤسسات البحثية مثل الجامعات من جهة، والشركات التي تمول المشروعات البحثية من جهة أخرى. ويقوم هذا التعاون في الغالب على أساس عقود واتفاقات تتحكم في أولويات البحث العلمي والحرية الأكاديمية للباحثين والفرص المتاحة لنشر بكوثهم. فبدلاً من التركيز على البحوث الأساسية التي تسهم في تقدم العمل العلمي وتوظيف نتائجه في جهود الصحة الإنسانية والمحافظة على البيئة مثلاً، أصبحت جهود الباحثين تنصب على مساعدة الشركات الكبيرة على ترويج منتجاتا والحصول على أرباح عاجلة.

وفي مقالة نشرقا المجلة المتخصصة بقضايا التعليم العالي في أمريكا، تحدثت المقالة عن حالات تقوم بها شركات أدوية أمريكية بتجريب علاج معين على الناس في بلدان إفريقية، ويكون لذذا العلاج آثار جانبية تنتهي بموت المريض. وعندما يكتشف أحد العلماء هذه الحقيقة، تسارع الشركة المنتجة للدواء بإخفاء الأدلة على خطورة العلاج، وتمارس الترغيب والترهيب على الجهات التي تنتقد استخدام العلاج، ولتقوية موقفها تستخدم الشركة المال للحصول على مساعدة الجامعات، فيتصدى بعض الأساتذة الجامعيين المرموقين لنشر بحوث في ججالت مرموقة لصالح العلاج، دون الإشارة إلى أن البحوث قامت بها الشركة المنتجة للدواء. أما الباحثة التي اكتشفت العلاج فيتم منحها موقعاً مهما في أحد المستشفيات يدرُّ عليها دخلاً مالياً كبيراً، وتكون تحت المراقبة حتى تبقى صامتة. وعندما تضطر إلى الاعتراف بالحقيقة، يتم تشويه سمتها وتتخذ جامعتها التي تتلقى تبرعات مالية من الشركة المنتجة- موقفاً ضدها، كما يفعل المستشفى - الذي يتلقى تبرعات مالية من الشركة نفسها- موقفاً مماثلاً.

وفي قصة مشابهة يكتشف تايرون هيز Tyron Hayes أحد علماء جامعة كاليفورنيا- بيركلي أن عقاراً معيناً من مضادات الحشرات يسبب إيذاء للتوازن البيئي، ويظن أن اكتشافه هذا سيكون مثيرا ومهماً، ولم يتخيل عدم الحماس التي تلقت به المؤسسة الداعمة لبحوثه النتائج التي توصل إليه. وبعد مضي ست سنوات وهو يواصل البحث ويؤكد النتائج نفسها، يهين موعد بتحيد الترخيص للعقار من قبل الوكالة الأمريكية لحماية البيئة، وبتحد الشركة من يأتي ببحوث وبتحارب تؤكد موقفها وتدحض موقف الباحث. مع أن 
الباحث أيضاً يجد العديد من الباحثين الذين يؤكدون بالتجارب والبحوث العملية مصداقية نتائجه، ومع ذلك تعرض الشركة على "هيز" شراء صمته مقابل مليوني دولار. كما تعرض الشركة على جامعات أخرى تمويل بحوث حول الموضوع شريطة أن يكون للشركة- وليس للباحثين- حق نشر نتائج هذه البحوث! وقد نشرت بجلة American Scientist الأمريكية التي تصدرها جمعية البحوث العلمية في أمريكا وذلك في طبعتها الإلكترونية على الإنترنت- مقالة بعنوان المشكلات الأخلاقية في البحوث الأكاديمية، أشارت فيها إلى الدراسة المسحيّة التي قام بها ثلاثة من الباحثين، وقد طرحت نتائجها أسئلة مهمة حول المناخ الأخلاقي للتعليم العالي والبحث العلمي. وقد أشارت المقالة إلى أنه بالرغم من احتمال وجود العديد من الأمثلة على سوء التصرف ومخالفة أخلاقيات البحث- وبعض الأمثلة في غاية الخطورة- فإن عين الرقابة العامة قلما تفحصت هذه الحالات وأثارت الاهتمام بما.

واستطلعت هذه الدراسة آراء ألفين من طلبة الدكتوراه وألفين آخرين من أساتذة الكيمياء والهندسة المدنية والبيولوجيا الجزيئية وعلم الاجتماع، وذلك في أكبر أقسام هذه التخصصات في الجامعات الأمريكية وعددها 99 قسماً. وكان المطلوب من المشاركين في الدراسة أن يشيروا إلى خبراقم المباشرة حول عدد من الممارسات غير الأخلاقية في ممارسة البحث العلمي. وتوصل الباحثون إلى أن عدد حالات الممارسات غير الأخلاقية في البحث العلمي لا يمكن تجاهلها. وتشمل هذه الحالات أمثلة على السرقات العلمية وتزوير البيانات و"طبخ" النتائج، ووضع أسماء باحثين على بحوث منشورة لم يكن لهم أية إسهامات فيها، إضافة إلى الإهمال في تسجيل القياسات وحفظها في أثناء إجراء التجارب. وأن عدد هذه الحالات أكبر بكثير مما يمكن استنتاجه من عدد الحالات التي يتم الإعلان عنها وتنال اهتمام الرأي العام.

5 أزمة البحث العلمي في العلاقة بالسياسة والإدارة الحكومية في السنوات الأخيرة تواتر الحديث عن. دور الإدارة الأمريكية الحالية في عهد الرئيس جورج بوش (الابن) في امتهان كرامة العلم والعلماء، وسوء استعمال البحوث العلمية وتشويه نتائجها لتنسجم مع توجهات سياسية معينة أو لتستجيب لضغوط جماعات وشركات ذات مصالح اقتصادية. وارتفعت بعض الأصوات التي تستنكر هذه الممارسات. ففي 18 فبراير 2004 شارك أكثر من (60) عالماً -من الحاصلين على جوائز نوبل ومن الأطباء المشهورين ورؤساء 
الجامعات الكبيرة والمديرين السابقين لوكالات حكومية فيدرالية- في توقيع وثيقة مهمّة بعنوان: "الأمانة العلمية وصنع السياسات: استقصاء دور إدارة الرئيس بوش في سوء استعمال العلم." وقد أشارت الوثيقة إلى حالات عديدة قامت فيها إدارة الرئيس بوش بتجاوز المتطلبات النوعية الحناصة بالنظام الاستشاري في قضايا العلوم ومتطلبات استقلالية هذا النظام. وقد أثرت هذه التجاوزات على الروح المعنوية للعلماء العاملين في

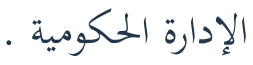

وذكرت الوثيقة أمثلة على علماء من ذوي الخبرات والكفاءات العالية استبعدت أسماؤهم من اللجان الاستشارية، وأمثلة أخرى على التحكم في عمل العلماء العاملين في الوكالات الحكومية، وبخاصة عندما تتصادم نتائج البحث العلمي مع السياسات الحكومية أو مع توجهات الفئات التي تقدم الدعم السياسي للحزب الجمهوري. وقد أهملت الإدارة الأمريكية الحالية تقارير علمية ذات أهمية بالغة قدمتها اللجان العلمية في مسائل عديدة.

وقد دعت الوثيقة إلى ضرورة وقف الجهود التي تشوه المعرفة العلمية لغايات سياسية حزبية، وإلى إتاحة

المجال للمواطنين أن يعرفوا الحقائق حول القضايا الأساسية التي قمم مصالحهم، والاستفادة من الأموال الضخمة التي تستثمر في البحث العلمي والتعليم، وإلى رفع مستوى الالتزام بالمعايير التي تتحكم في علاقة العلم بالحكومة، وأن على الكونغرس الأمريكي والإدارة التنفيذية عمل التشريعات اللازمة لمنع الرقابة السياسية على البحوث العلمية إلا لأسباب معقولة خاصة بالأمن القومي، إضافة إلى التأكيد على ضرورة امتلاك كك العلماء واللجان العلمية الرسمية أعلى المعايير المهنية الممكنة. وكان الهدف من التقرير هو توعية الرأي العام بخطورة الوضع والطلب إلى الحكومة العودة إلى الأخلاقيات ومعايير السلوك التي تدعم الاستقلالية والموضوعية في إسهامات العلم في بناء السياسات.

وقد أنشأ العلماء الذين وقعوا تلك الوثيقة اتحاداً للعلماء المهتمين بمتابعة هذه القضايا تحت اسم Union of Concerned Scientists نشرها إلى حوالي ستة آلاف عالم. وقد تم تحديث الوثيقة وإضافة كثير من التفاصيل ونشرها في كتاب . وقد جاءت في الكتاب تفصيلات توضح إجراءات الإدارة الأمريكية الحالية في كبت وتشويه نتائج البحث 
العلمي التي تتم في مختبرات ووكالات فيدرالية، وذلك فيما يتعلق بكثير من القضايا مثل: بحوث التغير المناخي، ونوعية الهواء الجوي، وقضايا الصحة الإنجابية، وقانون الأنواع الحية المهددة بالانقراض، وتحاليل البكتيريا الجوية، وبكوث التسمم بالرصاص، وقضايا الحماية والأمان في مواقع العمل، واستبعاد العلماء ذوي الخبرة والكفاءة العالية من اللجان الاستشارية في قضايا العلوم، وسوء استعمال العقاقير الطبية، وتسريح لجان الحد من التسلح النووي، ورفض الإصغاء إلى تقارير علمية موثوقة قدمتها يختبرات وطنية رسمية عديدة حول كذب الادعاءات الاستخبارية المتعلقة بمحاولة العراق الحصول على أنابيب ألومنيوم لازمة لتخصيب اليورانيوم،... وغيرها. وكشفت الدراسة التفصيلية عن جهود الإدارة الأمريكية في تحديد أسماء العلماء الذين يمكنهم تحكيم بعض البحوث للاطمئنان إلى أن نتائج هذه البحوث ستكون لصالح شركات ومؤسسات منتفعة منها، هذا فضلاً عن تشويه البيانات العلمية بحذف فقرات ذات دلالات معينة من نتائج البحوث العلمية وتقاريرها، وعلى عدم الامتثال للتوصيات المهمة التي تتوصل إليها هذه البحوث.

خاتمة

لم يكن الغرض من عرض هذه المؤشرات استقصاء مشكلات البحث العلمي في بلداننا الإسلامية أو في

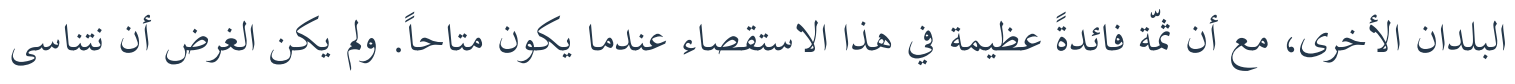
أن ممارسات البحث العلمي في السابق قد خلت من المشكلات، ولو أن المشكلات في السابق كانت تتعلق في الغالب بأخطاء فرادى الباحثين وتوجهاعم الإيديولوجية، بينما يتبدى وجه الخطورة أن المشكلات اليوم تتعلق بمؤسسات ضخمة وقوى هائلة.

وإنما كان الغرض التأكيد على أنّ البلدان القوية والغنية استطاعت -بما تملكه من مؤسسات علمية تنفق فيها مبالغ طائلة على إجراء البحث العلمي ونشره واستثماره، وبما تملكه من وزن اقتصادي ونفوذ سياسي- أن تجعل البلدان الأخرى تابعة لها ومعتمدة عليها في البحث العلمي والمشورة العلمية. فإذا كان ثمة ما يبرر الشك في بحوث تلك البلدان المتقدمة، وإذا كانت ظروف البحث في البلدان النامية على ما هي عليه من ضعف في إمكانيات التمويل والتنفيذ والتوظيف، أدركنا حجم الخطر الذي يتهدد بلادنا نتيجة مشكلات 
البحث العلمي عندنا وعند الآخرين، وأدركنا حجم الحاجة إلى تضافر جهود الباحثين في قضايا المنهجية، من التخصصات والخبرات المختلفة، بحيث تتكامل هذه الجهود من أجل بناء نسق معرفي يحكم مناهج البحوث وتطوير الأدوات الضرورية لتوظيف هذه المناهج في الخصول على المعرفة واختبارها وتطبيقها. وربما تكفي الإشارة في هذا العجالة إلى صورة واحدة من صور الأزمة التي نواجهها في البحوث المتعلقة بالعلوم الإسلامية، وفرص تفعيل الرؤية التي تقدمها هذه العلوم في مجالات العلوم الأخرى، وهي صعوبة تكوين فرق البحث حين يلزم تكوينها، فإذا كان التكامل بين العلوم الإسلامية والعلوم الأخرى مثلاً، سواءً كانت اجتماعية إنسانية أو طبيعية كونية هدفاً من أهداف إسلامية المعرفة فإن تعصب أصحاب هذه التخصصات لتخصصاتم كان عقبة رئيسية حالت دون نجاح برامج التعاون والتكامل بين الفريقين في بعض الأحيان. ومعظم برامج ومشاريع البحث في التأصيل الإسلامي وإسلامية المعرفة كانت تقترح أن يتم العمل من خلال فرق الباحثين، حيث يتكون الفريق من متخصصين في العلوم الإسلامية ومتخصصين في العلوم الاجتماعية المعاصرة ذوي العلاقة بموضوع البحث .

ولا شك في أن أفراداً عديدين من الفريقين يقدرون حاجتهم إلى الاستفادة من فهم الفريق الآخر وخبرته، لكن أفراداً آخرين يُنْكِرون حق غير المتخصص في محاولة الفهم والاجتهاد في غير اختصاصه. وإذا كان ذلك عيباً في علاقات بعض أهل التخصصات العلمية فهو في الدائرة الإسلامية أكثر عيباً سواءً في حق ورد المتخصصين في العلوم الإسلامية أو غيرهم. وقد يتجاوز الأمر حد إنكار المتخصص في علوم الحديث مثلاً أن يجتهد في فهم هذا العلم زملاؤه المتخصصون في علوم القرآن أو الفقه... مع العلم أن الفارق في خبرة هؤلاء المتخصصين جميعاً ربما لا يتجاوز أطروحة الدكتوراه التي أعدها الباحث وقد لا يتجاوز جهوده في هذه الأطروحة أكثر من تحقيق مخطوط!

ومن المؤسف حقاً أن التخصص في العلوم الإسلامية أصبح في بعض الأحيان مهنة وحرفة، مع العلم بأن الملاحظ في واقع الحياة أن كثيراً من غير المتخصصين في العلوم الإسلامية لا يقلون عن هؤلاء المتخصصين في قدرقم على فهم قضايا الدين أو الإفتاء في بعض المسائل أو ممارسة الدعوة والتدريس في المسائل الدينية. ذلك أن التدين والرغبة في التفقه في الدين لم تكن في الدائرة الإسلامية تخصصاً في يوم من الأيام. وعلى الله قصد السبيل. 